\title{
Combien de temps les élèves du primaire et du premier cycle du secondaire passent-ils en classe?
}

- Dans les pays de l'OCDE, les élèves sont censés suivre un total de 7751 heures d'instruction, en moyenne, durant leur scolarité dans le primaire et le premier cycle du secondaire ; la majeure partie de ce temps d'instruction est obligatoire.

- En règle générale, le nombre annuel d'heures d'instruction augmente avec le niveau d'enseignement.

- La lecture, les mathématiques et les sciences représentent environ $\mathbf{5 0} \%$ du temps d'instruction obligatoire dans le primaire, contre seulement $\mathbf{4 0} \%$ dans le premier cycle du secondaire.

- La forte variation du temps d'instruction entre les pays de l'OCDE laisse penser qu'on est loin d'avoir trouvé un consensus concernant les politiques les plus efficaces en matière de temps scolaire.

Le nombre d'heures passées en classe varie sensiblement entre les pays.

Dans les pays de I'OCDE, le temps d'instruction total prévu s'établit à 7751 heures, en moyenne, durant la scolarité dans le primaire et le premier cycle du secondaire, mais ce chiffre masque d'importantes variations entre les pays. Le temps total d'instruction réglementaire va de 6054 heures en Hongrie à 10710 heures en Australie. Durant ces heures, les établissements d'enseignement offrent une instruction dans les matières obligatoires du programme et, le cas échéant, dans des matières non obligatoires. Toutefois, la part du temps d'instruction consacrée aux matières non obligatoires est relativement limitée (voir la figure 1).

Figure 1. Nombre d'heures d'instruction prévues dans les établissements publics (2011)

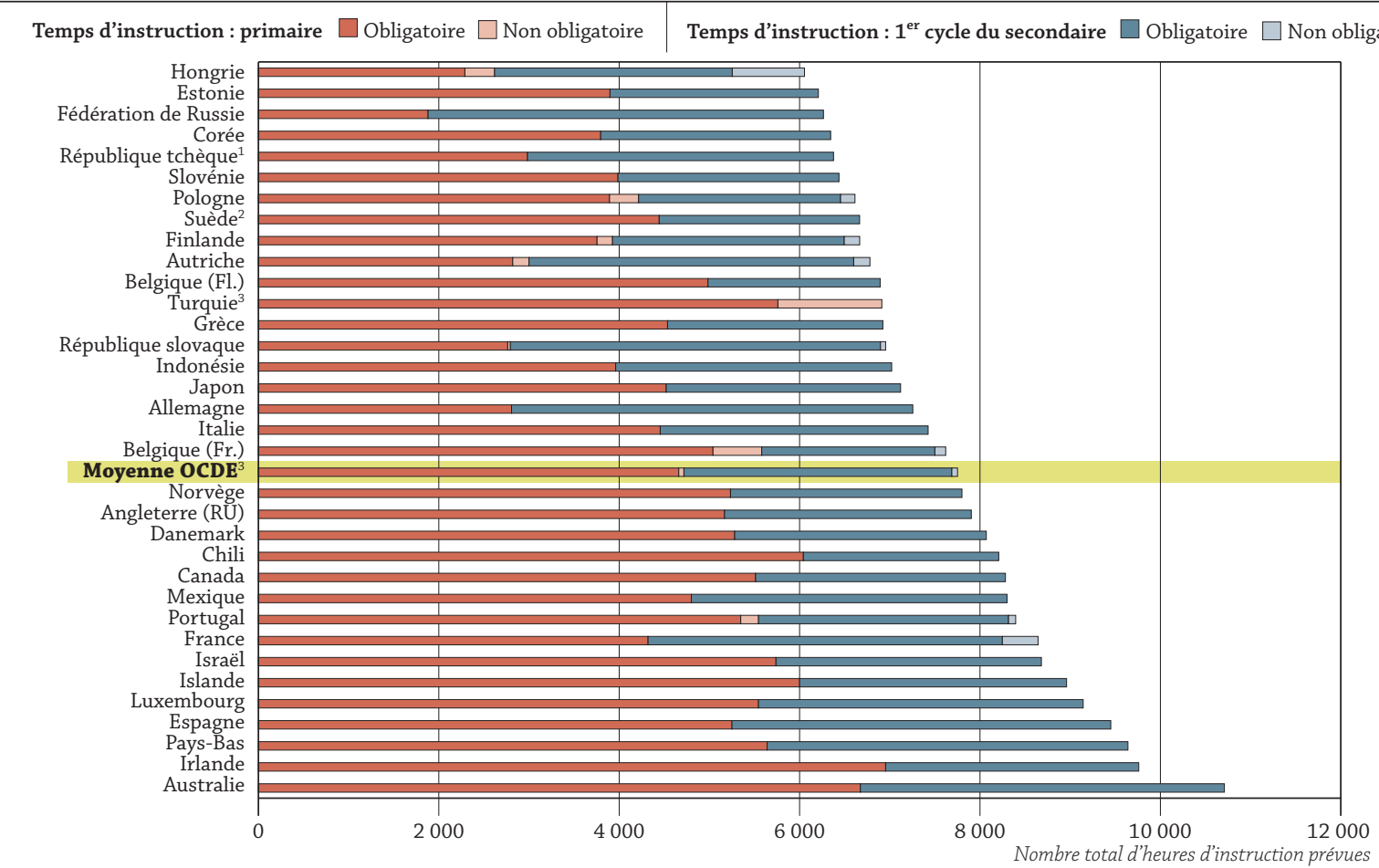

Remarque : par temps d'instruction prévu, on entend le nombre annuel d'heures que la réglementation publique prévoit pour l'instruction des élèves. Par programme obligatoire, on entend le temps d'instruction et sa répartition qui s'appliquent à la quasi-totalité des établissements d'enseignement publics. Par partie non obligatoire du programme, on entend le nombre moyen d'heures d'instruction supplémentaires que les élèves peuvent suivre en sus du temps d'instruction obligatoire.

1. Nombre minimum d'heures par an.

2. Estimation du nombre minimum d'heures par an en raison de l'absence de données ventilées par âge.

3. La Turquie n'est pas incluse dans la moyenne.

Les pays sont classés par ordre croissant du nombre total d'heures d'instruction prévues.

Source : OCDE (2013), Regards sur l'éducation 2013 : Les indicateurs de l'OCDE, indicateur D1 (www.oecd.org/edu/rse.htm). 
Dans les pays de l'OCDE, le temps annuel d'instruction prévu s'établit à 802 heures, en moyenne, pour les élèves du primaire. II ne passe la barre des 900 heures qu'en Australie, au Canada, au Chili, en Communauté française de Belgique, en Israël, au Luxembourg, aux Pays-Bas et au Portugal. Dans le premier cycle du secondaire, le temps annuel d'instruction prévu est supérieur de 122 heures, en moyenne, à celui du primaire. Cet écart passe

la barre des 200 heures en Corée, en Finlande, en France, en Hongrie, au Mexique et en République tchèque.

La lecture, l'expression écrite et la littérature, ainsi que les mathématiques et les sciences, représentent la moitié du programme obligatoire du primaire.

La part du temps d'instruction consacrée aux différentes matières du programme du primaire et du premier cycle du secondaire varie entre les pays de l'OCDE (voir la figure 2). Certaines tendances se dessinent néanmoins. À ces deux niveaux d'enseignement, environ trois quarts du temps d'apprentissage sont consacrés à six matières : la lecture, l'expression écrite et la littérature ; les mathématiques ; les sciences ; les sciences sociales ; les langues vivantes étrangères ; et les disciplines artistiques.

Figure 2. Répartition du temps d'instruction par matière du programme obligatoire du primaire et du premier cycle du secondaire, moyenne OCDE (2011)
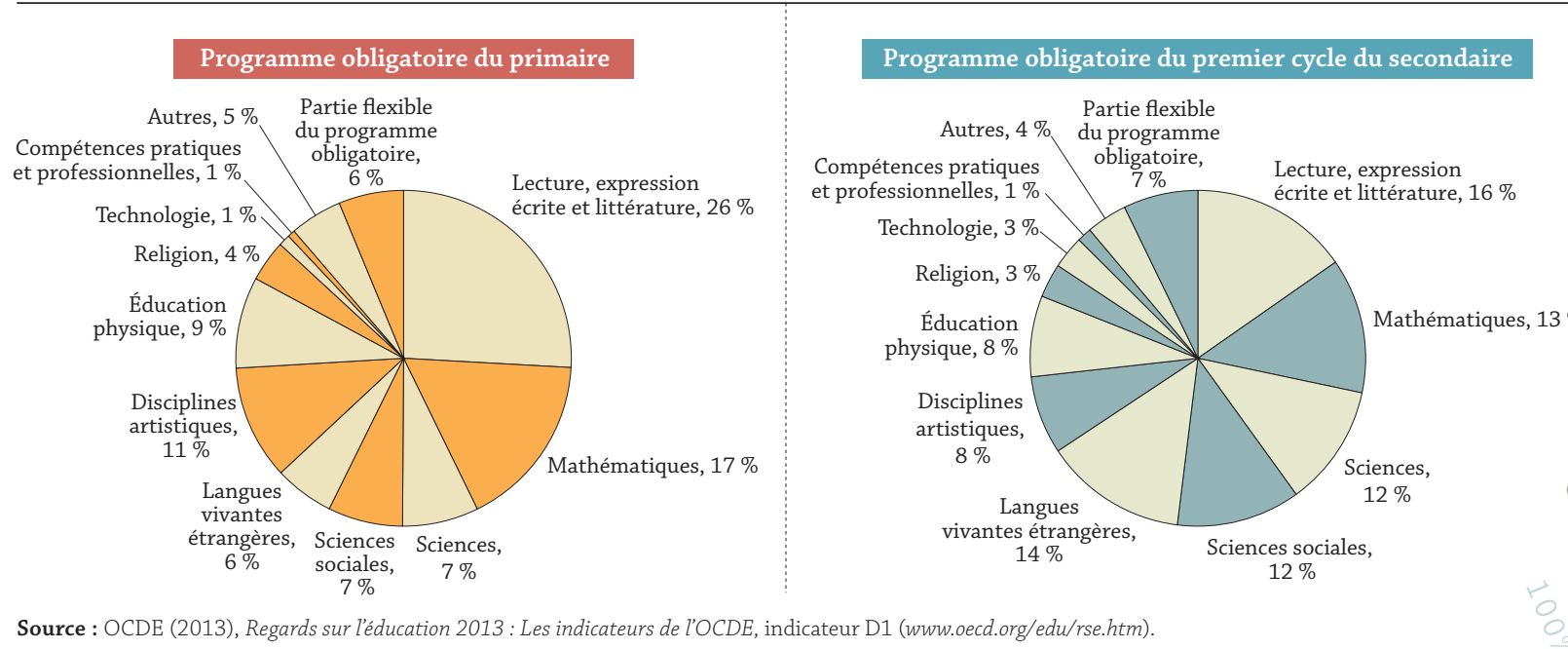

Source : OCDE (2013), Regards sur l'éducation 2013 : Les indicateurs de l'OCDE, indicateur D1 (www.oecd.org/edu/rse.htm).

Dans le primaire, environ $50 \%$ du temps d'instruction consacré au programme obligatoire sont dédiés à trois matières : la lecture, l'expression écrite et la littérature ; les mathématiques; et les sciences. Dans certains pays, la part du temps d'instruction consacrée à ces matières est moindre, s'établissant par exemple à seulement $38 \%$ en Allemagne et à $40 \%$ en Islande, tandis que dans d'autres, elle est sensiblement plus élevée (au Mexique, ces trois matières représentent 77 \% du programme du primaire). Dans le primaire, la lecture, l'expression écrite et la littérature sont la matière à laquelle on accorde la plus grande priorité (en moyenne, un quart du temps d'instruction). Les élèves du primaire consacrent plus d'un tiers de leur temps d'instruction à cette matière au Mexique (35\%), en France (37\%) et en Hongrie (38\%).

\section{Les langues vivantes étrangères, les sciences et les sciences sociales occupent une place} plus importante dans le premier cycle du secondaire.

Dans le premier cycle du secondaire, l'ordre de priorité des différentes matières commence à changer. Tandis que le temps moyen d'instruction consacré à la lecture, l'expression écrite et la littérature diminue, passant de $26 \%$ à $16 \%$, celui consacré aux langues vivantes étrangères augmente, passant de $6 \%$ à $14 \%$.

De même, la plupart des pays consacrent au moins deux fois plus de temps d'instruction aux mathématiques qu'aux sciences dans le primaire, alors que dans le premier cycle du secondaire, la part du temps d'instruction consacrée à ces deux matières s'équilibre. Là encore, la répartition réelle varie, certains pays continuant à privilégier les mathématiques (comme la Communauté flamande de Belgique ou l'Italie), tandis que d'autres consacrent davantage de temps aux sciences (l'Estonie et la Finlande, par exemple). 


\section{INDICATEURS DE L'ÉDUCATION À LA LOUPE}

\section{Tandis que les disciplines artistiques et l'éducation physique continuent à occuper une certaine place dans le programme obligatoire, la part du temps d'instruction consacrée à la technologie est relativement faible.}

Dans les pays de l'OCDE, le programme des disciplines artistiques est en général axé sur les arts visuels, la musique, le théâtre et la danse (Winner et al., 2013). La part du temps d'instruction consacrée aux disciplines artistiques varie entre $5 \%$ et $20 \%$ du programme obligatoire dans le primaire. Dans le premier cycle du secondaire, la plupart des pays y consacrent moins de temps, à quelques exceptions près, comme l'Autriche. Les disciplines artistiques étant souvent proposées à titre d'option dans le premier cycle du secondaire, certains élèves peuvent en fait y consacrer plus de temps dans les classes supérieures.

Dans les pays de l'OCDE, la part du temps d'instruction consacrée à l'éducation physique est assez similaire dans le primaire et le premier cycle du secondaire (respectivement $9 \%$ et $8 \%$ ).

La part du temps d'instruction consacrée à la technologie est relativement faible à ces deux niveaux d'enseignement ( $1 \%$ dans le primaire et $3 \%$ dans le premier cycle du secondaire). Cette part est toutefois plus importante dans quelques pays, tels que le Chili (7 \% dans le primaire), I'Italie et l'Angleterre (Royaume-Uni) (respectivement $7 \%$ et $13 \%$ dans le premier cycle du secondaire). Par ailleurs, même lorsqu'elle n'est pas considérée comme une matière à part entière du programme obligatoire, la technologie pourrait être utilisée comme outil d'apprentissage dans d'autres matières.

La durée de l'année scolaire varie aussi sensiblement entre les pays.

Les pays doivent non seulement décider du nombre d'heures d'instruction, mais également de leur répartition au cours de l'année et de la journée scolaires de façon à optimiser les résultats de l'apprentissage. Une journée scolaire trop longue peut induire un temps de rattrapage trop limité pour les élèves en difficulté, alors que la répartition du même nombre total d'heures sur un plus grand nombre de semaines d'école offre davantage de flexibilité pour l'organisation de la journée scolaire et peut permettre de proposer des séances de soutien individuel à des moments plus productifs.

Dans certains pays, le nombre de semaines d'enseignement est inférieur à la moyenne, impliquant des journées scolaires plus denses pour les élèves (comme en Belgique, au Canada, en Espagne, en France, en Irlande, en Islande et au Luxembourg) ; dans d'autres (comme en Australie, en Italie et aux Pays-Bas), le temps d'instruction est similaire, mais réparti sur un plus grand nombre de semaines d'école, permettant d'avoir des journées scolaires moins chargées (voir la figure 3).

La forte variation entre les pays des deux variables présentées à la figure 3, ainsi que leur faible corrélation, laissent penser qu'on est loin d'avoir trouvé un consensus sur les politiques les plus efficace en matière de temps d'instruction.

Figure 3. Nombre annuel d'heures d'instruction prévues et nombre annuel de semaines d'enseignement dans le primaire (2011)

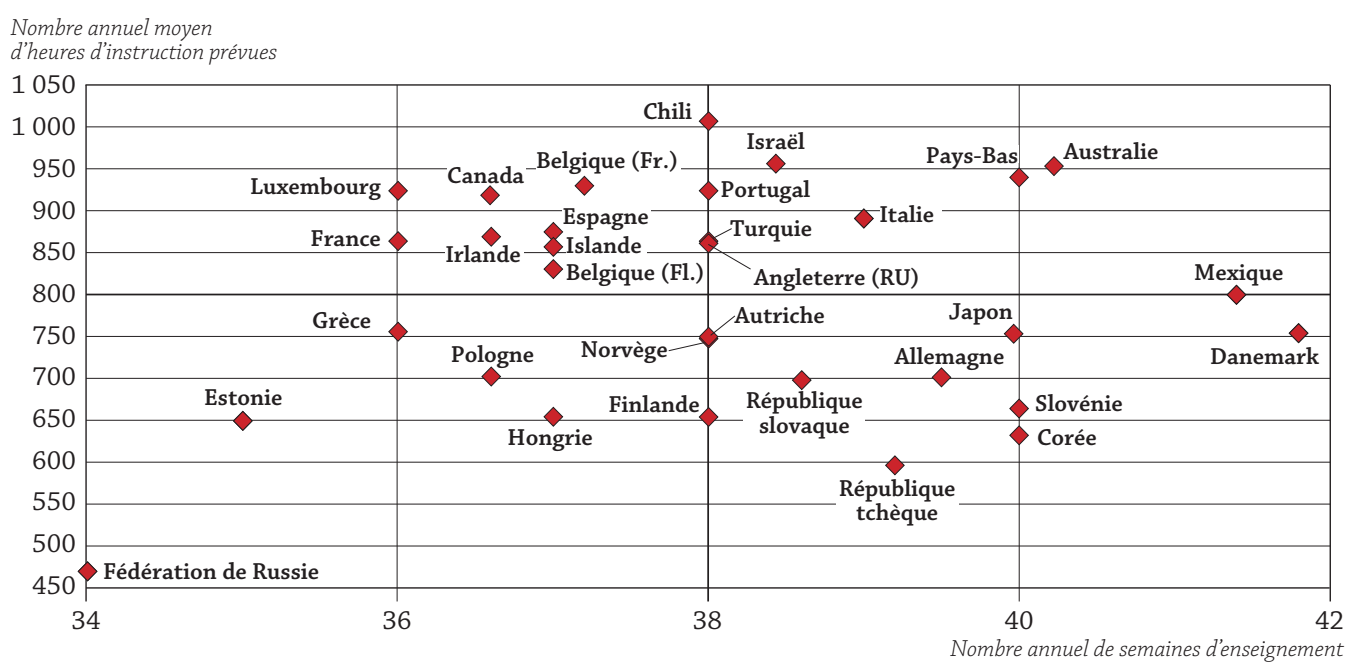

Source : OCDE (2013), Regards sur l'éducation 2013 : Les indicateurs de l'OCDE, indicateurs D1 et D4 (www.oecd.org/edu/rse.htm). 


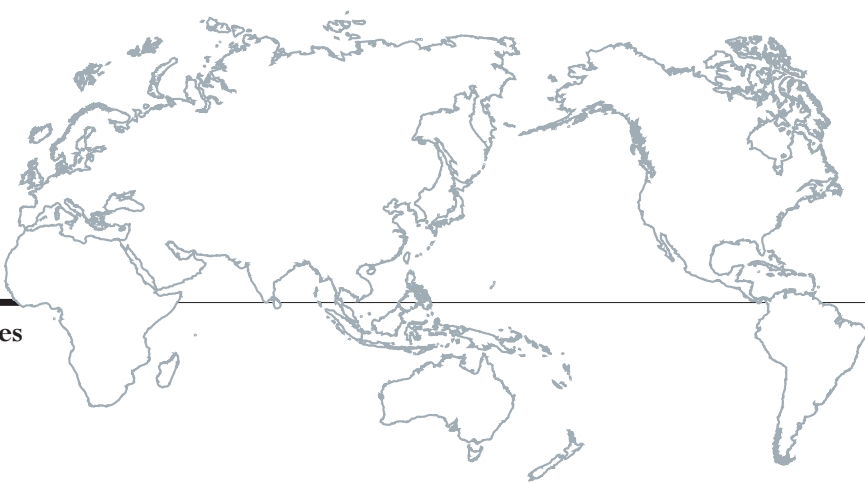

Les élèves peuvent également, pour différentes raisons, passer du temps supplémentaire à étudier en dehors du cadre scolaire.

S'il est instructif de réfléchir au nombre d'heures consacrées à l'apprentissage à l'école, le temps passé à étudier en dehors du cadre scolaire doit également être pris en considération, qu'il prenne la forme de devoirs, d'activités extrascolaires ou de cours supplémentaires en dehors de l'école (OCDE, 2013c).

Aucun lien n'a toutefois été clairement établi entre les résultats scolaires et le temps consacré aux cours extrascolaires et à l'étude individuelle. Par exemple, d'un côté, il n'est pas d'usage en Finlande de consacrer du temps supplémentaire à étudier en dehors de l'école, mais ce pays est l'un des plus performants en matière d'éducation, et de l'autre, en Corée et au Japon, les élèves passent de nombreuses heures à étudier dans des cours privés du soir, et ces pays obtiennent souvent des résultats élevés dans les évaluations internationales par rapport à de nombreux autres pays de l'OCDE.

\section{En matière de temps d'instruction, la quantité compte moins que la qualité.}

Le nombre d'heures passées à l'école compte bien moins que la façon dont ces heures sont utilisées, les matières auxquelles elles sont consacrées, les méthodes d'enseignement et d'apprentissage mises en œuvre, le contenu du programme et la qualité des enseignants. Certaines études se sont ainsi intéressées à des méthodes novatrices concernant I'utilisation et la répartition du temps d'enseignement à l'école visant à optimiser les possibilités d'apprentissage (OCDE, 2013b).

Entre 2005 et 2011, le nombre d'heures d'instruction a diminué de $3 \%$, en moyenne, dans le primaire (d'après l'estimation du nombre d'heures d'instruction des 9-11 ans), mais est resté stable dans le premier cycle du secondaire (d'après l'estimation du nombre d'heures d'instruction des 12-14 ans). En particulier, le temps total d'instruction prévu dans le primaire a diminué en Autriche, en Estonie, en Grèce et en Hongrie. En Estonie, le volume des matières obligatoires au titre de l'éducation de base est également passé de 72 à 63 cours, et davantage de cours sont désormais proposés à titre d'option.

Dans d'autres pays, tels que la Norvège, la Pologne et le Portugal, le temps total d'instruction prévu a augmenté entre 2005 et 2011, avec pour principal objectif de revaloriser la place de la lecture et des mathématiques dans le programme.

Pour conclure : Le nombre d'heures d'instruction que les élèves sont censés recevoir varie sensiblement entre les pays, mais il compte moins que la façon dont ces heures sont utilisées, les matières auxquelles elles sont consacrées et le contenu du programme. La lecture et les mathématiques représentent la majorité du temps d'instruction dans le primaire, tandis que la part de ce temps consacrée aux sciences, aux sciences sociales et aux langues vivantes étrangères augmente dans le premier cycle du secondaire. La technologie n'est, quant à elle, guère présente à ces deux niveaux d'enseignement.

\section{Pour plus d'informations}

OCDE (2013a), Regards sur l'éducation 2013 : Les indicateurs de l'OCDE, Éditions OCDE, Paris.

OCDE (2013b), Innovative Learning Environments, La recherche et l'innovation dans l'enseignement, Éditions OCDE, Paris.

OCDE (2013c), PISA 2012 Results: What Makes Schools Successful (Volume IV): Resources, Policies and Practices, PISA, Éditions OCDE, Paris (à paraitre en français).

Winner, E., T. Goldstein et S. Vincent-Lancrin (2013), Art for Art's Sake? The Impact of Arts Education, La recherche et l'innovation dans l'enseignement, Éditions OCDE, Paris.

\section{Contacter}

Eric Charbonnier (Eric.Charbonnier@oecd.org) / Nhung Truong (Nhung.Truong@oecd.org)

$\begin{array}{ll}\text { Voir } & \begin{array}{l}\text { Le mois prochain } \\ \text { www.oecd.org/edu/rse.htm/ }\end{array} \\ \begin{array}{l}\text { À quel âge les étudiants de l'enseignement tertiaire obtiennent-ils } \\ \text { leur premier diplôme? }\end{array} \\ \underline{\text { PISA à la loupe }}\end{array}$

Crédit photo : @ Ghislain \& Marie David de Lossy/Cultura/Gett y Images

Ce document est publié sous la responsabilité du Secrétaire général de l'OCDE. Les opinions qui y sont exprimées et les arguments qui y sont employés ne reflètent pas nécessairement les vues officielles des pays membres de l'OCDE.

Ce document et toute carte qu'il peut comprendre sont sans préjudice du statut de tout territoire, de la souveraineté s'exerçant sur ce dernier, du tracé des frontières et limites internationales, et du nom de tout territoire, ville ou région.

Les données statistiques concernant Israël sont fournies par et sous la responsabilité des autorités israéliennes compétentes. L'utilisation de ces données par l'OCDE est sans préjudice du statut des hauteurs du Golan, de Jérusalem-Est et des colonies de peuplement israéliennes en Cisjordanie aux termes du droit international. 